\title{
Treinos de Discriminação Simples e Formação de Classes Funcionais de Estímulos por Cães
}

\author{
Camila Domeniconi \\ Renato Bortoloti \\ Leilane Cristine Krutzfeldt Antoniazzi \\ Tássia Eid Nogueira Mendes \\ Universidade Federal de São Carlos
}

\begin{abstract}
RESUMO
O presente estudo verificou a formação de classes de estímulos funcionalmente equivalentes e learning set a partir de treinos de discriminações simples simultâneas e repetidas reversões nas funções dos estímulos, com uso de reforçadores diferentes e específicos para cada classe. Os sujeitos foram três cães sem raça definida. Nas tentativas de teste foram encontradas, em média, porcentagens de acertos de 93,6. Esses dados somados aos resultados das primeiras tentativas de apresentação de cada par de estímulos atestaram a reversão instantânea das funções de estímulos, ambos indicando a formação de classes funcionalmente equivalentes. Os dados de learning set não foram conclusivos a respeito da progressão na eficiência dos desempenhos nas reversões, possivelmente, por influência da preferência diferencial dos cães pelos reforçadores específicos utilizados em cada classe. $\mathrm{O}$ procedimento parece adequado e econômico para as investigações na área de comportamento simbólico em animais não humanos.
\end{abstract}

Palavras-chave: equivalência funcional; discriminações simples; reversões; reforço específico e diferencial; cães.

\begin{abstract}
Simple Discrimination Training and Formation of Functional Stimulus Classes by Dogs

This study attempted to verify the establishment of functional classes and learning set in dogs through a simple discrimination procedure with repeated reversals that included specific reinforcers for each stimulus class. Two stimuli were presented in each trial and the subject had to choose one of them by jumping in its direction. After being training in such procedure, three dogs responded correctly in more than $90 \%$ of the test trials. These performances and analysis of the first responses in the test sessions provided evidences of the establishment of functional stimulus classes. Data from the repeated reversals training did not provided evidences of the establishment of a learning set by the dogs. The discussion examines whether the non-establishment of a learning set could be influenced by preferences for specific reinforcers. The procedure seems adequate and economical in investigations on symbolic potential of nonhuman animals.
\end{abstract}

Keywords: functional equivalence; simple discrimination; reversals; differential and specific reinforcement; dogs.

O procedimento de reforçar respostas específicas somente diante de um estímulo designado como "positivo" e de não reforçá-las quando um estímulo "negativo" é apresentado pode ser suficiente para ensinar uma discriminação simples. Se o ensino for efetivo, o sujeito passará a emitir as respostas reforçadas diante do estímulo positivo e a não emiti-las, ou emiti-las raramente, diante do estímulo negativo (de Rose, Mcllvane, Dube, Galpin, \& Stoddard, 1988). Discri- minações condicionais podem ser estabelecidas a partir do ensino de relações entre um conjunto de dois ou mais estímulos apresentados como modelo (e.g., A1, A2,... An) e um conjunto de dois ou mais estímulos de comparação (e.g., B1, B2,... Bn). Por exemplo, na presença do modelo A1 e dos estímulos de comparação B1, B2 e B3, somente a escolha do estímulo de comparação B1 é reforçada. Se o ensino for efetivo, o sujeito passará a emitir respostas de seleção de B1 
condicionalmente à apresentação de A1. Sidman e Tailby (1982) demonstraram que a aprendizagem de discriminações condicionais pode dar origem a relações emergentes (relações que não são ensinadas diretamente, mas derivadas do treino discriminativo), que atestariam a formação de classes de estímulos equivalentes com propriedades simbólicas (ver também Sidman, 1994, 2000).

Vaughan (1988) foi pioneiro na demonstração de desempenhos emergentes que atestariam a formação de classes de estímulos funcionalmente equivalentes geradas a partir de discriminação simples. Utilizando pombos como sujeitos, esse estudo envolveu 40 estímulos visuais que eram apresentados em séries de tentativas sucessivas de discriminação simples. Em cada tentativa era apresentado um estímulo, o pombo deveria bicar os estímulos que foram designados como positivo (em um subconjunto de 20) e não bicar os designados como negativo (os outros 20 estímulos). Após o treino de vários pares, os estímulos tinham as suas funções revertidas, ou seja, os estímulos originalmente positivos passavam a ser negativos e os estímulos originalmente negativos passavam a ser positivos para a resposta de bicar. Atingidos os critérios de aprendizagem, as funções eram novamente revertidas. Depois de repetidas reversões, Vaughan (1988) observou que, quando um estímulo tinha a sua função discriminativa revertida, os outros estímulos envolvidos na mesma história de reforçamento passavam a ter as suas funções também revertidas, ainda que sem treino explícito. Assim, por meio de um treinamento de discriminações simples, Vaughan demonstrou que pombos se tornavam capazes de classificar um grande número de estímulos em dois conjuntos funcionalmente equivalentes. Ele argumentou que essas relações emergentes a partir de uma história de reforçamento partilhada entre os estímulos de cada conjunto confirmariam uma equivalência funcional entre os estímulos.

Adaptações do procedimento introduzido por Vaughan (1988) têm sido utilizadas em investigações mais recentes. Em uma delas, Lionello-DeNolf, Canovas, Souza, Barros e McIlvane (2008) realizaram dois estudos, um deles com crianças pré-escolares com desenvolvimento típico e o outro com adolescentes com autismo, utilizando procedimentos de discriminações simples simultâneas com repetidas reversões nas funções dos estímulos. Treinando três pares de estímulos separadamente (um par de cada vez) e intercalando esses pares em um treino misto, sempre revertendo as funções dos estímulos, os autores tiveram o objetivo de verificar ocorrências de learning set, caracterizado por desempenhos progressivamente mais eficientes nas reversões, e indícios de formação de classes de estímulos. Os resultados obtidos nos dois estudos mostraram alguns indícios claros de formação de classes de estímulos equivalentes pelas crianças com desenvolvimento típico e também resultados bastante promissores (embora com maior variabilidade) entre adolescentes com autismo, indicando que o procedimento pode representar um caminho interessante para o estudo da formação de classes por indivíduos com déficits de desenvolvimento.

O uso de treinos de discriminações simples visando à formação de classes funcionais de estímulos em não humanos tem sido descrito até o presente momento com leões marinhos (Kastak, Schusterman, \& Kastak, 2001) e com macaco prego (Goulart, Galvão, $\&$ Barros, 2003). No estudo de Kastak e colaboradores (2001) dois leões marinhos foram treinados a classificar 20 estímulos (letras e números) em dois subconjuntos de dez estímulos cada por meio de reversões sucessivas nas funções discriminativas dos estímulos. Os experimentadores utilizaram reforçamento diferencial e específico para cada classe de estímulos treinada. Os dois sujeitos demonstraram indícios da formação de classes funcionais após seis fases experimentais contidas no primeiro estudo. Discutiu-se posteriormente a possibilidade de explorar empiricamente o potencial dos treinos de discriminação simples com reversões sucessivas nas funções discriminativas dos estímulos, de forma a obter com eficiência a formação de classes funcionais em menos sessões e com menos reversões do que fizeram Kastak e colaboradores e Vaughan.

No estudo de Goulart e colaboradores (2003) foram realizados treinos de discriminações simples simultâneas e repetidas reversões nas funções discriminativas dos estímulos com um macaco prego em três experimentos. Foram treinados três conjuntos de dois estímulos cada, apresentados por meio de um micro computador acoplado à caixa experimental. Os dados obtidos por Cebus apella no estudo de Goulart e colaboradores (2003) não foram conclusivos na direção de formação de classes funcionais. Os autores sugeriram que procedimentos de discriminação simples com reversões sucessivas poderiam ser adequados para as investigações propostas e que alterações, como a inserção de reforçamento específico e diferencial para cada classe treinada (como foi realizado por Kastak e colaboradores, 2001) poderia aumentar as possibilida- 
des de controle por aspectos relevantes dos estímulos facilitando a formação de classes funcionais.

O presente trabalho teve o objetivo geral de investigar a formação de classes de estímulos por cães a partir de um procedimento de discriminações simples simultâneas com reversões sucessivas nas funções dos estímulos e o uso de reforço específico e diferente para cada classe de estímulo. A formação de classes de estímulos foi investigada por meio de registros de reversão espontânea da função de um estímulo após a exposição às contingências com estímulos da mesma classe e ocorrências de learning set, caracterizado por desempenhos progressivamente mais eficientes nas reversões.

O cão tem sido apontado como particularmente interessante para estudos comportamentais porque, integrado ao ambiente humano há milhares de anos, trata-se de um animal que desenvolveu uma sofisticada habilidade de responder a estímulos apresentados pelo homem (Udell \& Wynne, 2008).

\section{MÉTODO}

\section{Sujeitos}

Foram sujeitos do experimento três cães do sexo feminino, de raça indefinida e com idades entre um e três anos (de acordo com estimativa veterinária). Os animais - Tobi, Nina e Duquesa - viviam em situação de cativeiro no canil municipal da cidade de São Carlos.

\section{Local}

As sessões experimentais ocorriam nas dependências do próprio canil, em uma sala de 3,0 x 1,5 m que dispunha de razoável isolamento sonoro e de outras interferências externas. Os animais eram retirados do viveiro que habitavam e conduzidos à sala de coleta de dados apenas nos dias em que havia sessões. Antes ou depois das sessões, os experimentadores conduziam os sujeitos para um passeio pela área aberta do canil a fim de diminuir um pouco a atividade motora do animal (em caso de passeios que ocorriam antes da sessão experimental) ou reforçar a participação deles (em caso dos passeios que ocorriam depois das sessões).

\section{Situação e materiais}

Como estímulos foram utilizados pequenos utensílios domésticos ou brinquedos no interior dos quais se colocava uma porção de alimento. Ao escolher o estímulo correto, o animal tinha acesso ao alimento, os estímulos designados como incorretos não possuíam alimentos em seu interior. Cada par de estímulos estava correlacionado a um alimento específico.

No interior da sala experimental, o animal era colocado atrás de uma placa de madeira medindo $30,0 \mathrm{x}$ $60,0 \mathrm{~cm}$ (comprimento $\mathrm{x}$ altura) à qual se unia perpendicularmente outra placa de madeira de dimensões semelhantes, formando um aparato em forma de "T", conforme ilustrado na Figura 1.

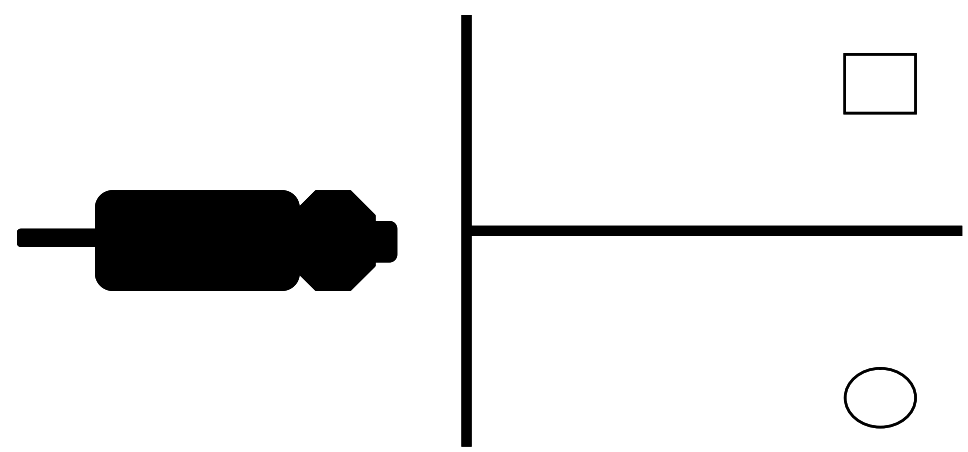

Figura 1. Representação do aparato experimental em forma de "T". O animal está representado esquematicamente ante uma barreira vertical. As figuras geométricas (círculo e quadrado) posicionadas no outro extremo do aparato representam os estímulos utilizados.

Cada sessão experimental era constituída por uma série de tentativas em que o sujeito devia escolher um de dois objetos que eram dispostos à sua frente. Após a disposição dos objetos e a visualização deles pelo sujeito, era permitido que o animal escolhesse um dos objetos apresentados saltando na direção dele. A tentativa era então encerrada com o acesso do animal ao reforçador (em caso de escolha do objeto designado experimentalmente como correto para aquela tentativa) e a recondução do mesmo para a posição inicial ou 
apenas a recondução (em caso de escolha do objeto designado como incorreto para aquela tentativa).

\section{Procedimento Geral}

Os sujeitos desta pesquisa foram escolhidos entre os animais de mais fácil manejo, conforme indicação das médicas veterinárias responsáveis pelo canil. Os animais selecionados ganharam uma coleira de couro padrão, na cor vermelha, para serem mais facilmente identificados pelos experimentadores e pelos funcionários do canil.

Os procedimentos de adaptação dos experimentadores ao trato com os sujeitos e dos sujeitos à rotina de pesquisa começaram com duas visitas ao canil, nas quais foram desenvolvidas as atividades de entrar no viveiro no qual estavam instalados os animais que participariam do trabalho, conectar as guias às coleiras de cada animal, sair com o animal de dentro do viveiro e passear com cada um deles individualmente. Estas visitas tiveram o objetivo de adaptar animais, experimentadores e funcionários com a entrada de pessoas estranhas no viveiro e a retirada de apenas alguns animais, um de cada vez.

Foi aplicado um teste de preferência com o objetivo de estabelecer os itens alimentares de preferência similar pelos animais, uma vez que o uso de reforçadores específicos e diferentes para cada classe de estímulo foi uma variável relevante para o experimento, ou seja, preferências muito diferentes por determinados reforçadores poderiam ocasionar um viés nos resultados. Foram utilizados oito diferentes itens alimentares apropriados para cães (salsicha, ração, biscoito canino básico, banana, "salaminhos", "bifinhos", pequenos itens imitando ossos com tutano e pequenos itens em formato de porções de carne). Os itens eram apresentados aos pares (totalizando 56 tentativas de escolha) com combinações entre estímulos e posições de apresentação de cada estímulo realizada de forma aleatória.

Os testes foram executados da seguinte maneira: em cada tentativa foram apresentadas ao animal as duas opções de cada par e permitia-se a ele fazer uma escolha e ingerir um dos itens. $\mathrm{O}$ item rejeitado de cada par apresentado não poderia ser consumido pelo animal. A aplicação do teste foi dividida em dois dias consecutivos a fim de não sobrecarregar o animal e também evitar qualquer possibilidade de saciar o animal, o que também poderia enviesar os resultados.

Modelagem da resposta alvo (saltar sobre o aparato em direção a um dos estímulos). Foram realiza- das três sessões com doze tentativas cada, com objetivos de modelar a resposta alvo esperada no animal (olhar para os dois objetos e saltar sobre o aparato em direção a um deles). $\mathrm{O}$ animal era conduzido até a porção superior do aparato e seu focinho era direcionado manualmente até que ele dirigisse o olhar a cada um dos objetos. Um dos experimentadores ficava posicionado junto ao animal e garantia sua posição no meio do aparato, permitindo a visualização igualitária dos dois lados do aparato e o outro se posicionava na base do aparato e se encarregava de posicionar os objetos e certificar que o animal havia olhado para ambos. Após a autorização deste experimentador a guia do animal era retirada permitindo a ele o salto sobre o aparato.

Os estímulos utilizados durante esta fase do procedimento foram os dois itens alimentares apontados como preferidos pelo animal durante o teste de preferência.

Durante a primeira sessão desta fase eram colocados estímulos nos dois lados do aparato simultaneamente, sendo assim, o salto a qualquer um dos lados do aparato possibilitava ao animal o acesso ao alimento em todas as doze tentativas. Nas tentativas iniciais, caso o animal não saltasse em cinco segundos o experimentador posicionado na base do aparato chamava o animal com um assobio ou estalar de dedos a fim de estimular o salto.

$\mathrm{Na}$ segunda sessão, apenas um dos lados do aparato continha um dos itens alimentares preferidos, desta maneira o animal tinha acesso ao alimento apenas se saltasse para o lado correto do aparato. Para maximizar as possibilidades de reforçamento, portanto, ele deveria olhar para os dois lados, discriminar aquele que continha alimento e saltar em direção a ele. Nesta sessão ainda poderia ter sido utilizado o chamado, caso o animal não se comportasse na direção de emitir a resposta alvo.

A terceira sessão foi idêntica à segunda, mas foi exigido o critério de $100 \%$ de saltos corretos (direcionados para o lado do aparato que continha o alimento) sem a utilização de nenhum chamado por parte do experimentador.

\section{Condições experimentais}

Linha de base - discriminações simples simultâneas e discriminações sucessivas com um par de estímulos de cada vez. Após a modelagem da resposta alvo e a seleção dos reforçadores, foram iniciadas as sessões para o estabelecimento da linha de base de 
discriminações simples simultâneas com reversões sucessivas e uso de reforço específico para cada classe.

Cada sessão foi composta por doze tentativas. Cada tentativa tinha início com o posicionamento do animal na porção superior do aparato, exatamente no meio dos dois lados, permitindo a visualização a ambos. Um dos experimentadores segurava o animal enquanto o outro posicionava o par de objetos (um em cada lado do aparato, sendo que apenas um deles continha um item alimentar dentro). Este experimentador posicionado na base do aparato procurava se certificar de que o animal havia olhado para ambos os objetos e então sinalizava ao outro experimentador que soltasse o animal. $\mathrm{O}$ animal era solto e deveria saltar sobre o aparato em direção a um dos lados.

Posicionados os objetos e o animal, caso a escolha do mesmo fosse a correta (ou seja, caso ele saltasse para o objeto que continha o alimento em seu interior) o experimentador passava a mão sobre a cabeça do animal e o auxiliava na abertura do objeto, permitindo a ele a ingestão do alimento. Após ingerir o alimento ele era reconduzido para a posição inicial e uma nova tentativa era iniciada. Caso a escolha do animal fosse incorreta (saltando ele em direção ao objeto que não continha alimento), o experimentador o auxiliava a abrir o objeto e ele era reconduzido para a posição inicial, recomeçando uma nova tentativa.

Os pares de objetos eram apresentados simultaneamente (discriminação simples simultânea) e apenas um deles de cada vez estava correlacionado com o reforço. Após o alcance do critério de aprendizagem a relação era revertida, ou seja, o objeto anteriormente negativo (não correlacionado ao reforço) passava a ser positivo (correlacionado ao reforço) e vice-versa. O critério de aprendizagem para todos os pares foi de $90 \%$ de acerto em três sessões consecutivas. Todas as relações foram revertidas três vezes, ou seja, cada objeto de cada par funcionou duas vezes como estímulo positivo e duas vezes como estímulo negativo.

O estabelecimento da linha de base teve início com a apresentação dos pares A1 e B1, sendo que o estímulo A1 foi experimentalmente definido como correto e relacionado ao reforçador RA (salsicha). Depois de atingido o critério de aprendizagem a relação foi revertida, ou seja, B1 passou a ser estímulo discriminativo e a seleção dele foi reforçada com a apresentação ao reforçador RB (salaminho). A função deste mesmo par de objetos foi revertida mais duas vezes, ou seja, A1 funcionou como estímulo discriminativo, correlacionado ao RA, duas vezes e B1 funcionou como estímulo discriminativo, correlacionado ao RB, também duas vezes. $\mathrm{O}$ mesmo ocorreu com os pares A2/B2 e A3/B3.

Importante ressaltar que, durante o estabelecimento da linha de base, em cada sessão era utilizado apenas um mesmo par de objetos e as funções deles também nunca foram revertidas dentro de uma mesma sessão.

Testes. Foram programados blocos de teste estruturados com tentativas utilizando objetos diferentes, em uma mesma sessão. A estrutura geral dos testes foi a mesma descrita acima com uma alteração: as doze tentativas consistiram em quatro tentativas com a utilização de cada par de objetos, ou seja, quatro tentativas com $\mathrm{A} 1$ e $\mathrm{B} 1$, quatro com $\mathrm{A} 2$ e $\mathrm{B} 2$ e quatro com A3 e B3.

Os critérios de acerto foram cancelados e as reversões ocorreram simultaneamente para todos os membros dos três conjuntos, ou seja: em um bloco de treino, os estímulos A1, A2 e A3 funcionavam como estímulos discriminativos e estavam correlacionados com o RA (salsicha). Após uma sessão completa todas as funções dos estímulos eram revertidas, assim, B1, B2 e B3 passavam a estar correlacionados com o RB (salaminho). As reversões nas funções dos objetos nunca ocorreram em uma mesma sessão.

Foram realizadas outras duas sessões de testes, visando tornar ainda mais aleatória a apresentação dos objetos. A primeira delas consistiu na apresentação alternada dos três pares de objetos, sendo que funcionavam como estímulos discriminativos os objetos correlacionados com o R1 (salsicha), ou seja, A1, A2 e A3. A sessão teve início com a apresentação do primeiro par de estímulos, A1 e B1, durante duas tentativas. Na sequência, foram apresentados A2 e B2 (2 tentativas) e A3 e B3 (2 tentativas). A sétima tentativa, consistiu na apresentação de A1 e B1 novamente. Até a décima segunda tentativa foram alternados os pares de objetos. A quantidade de apresentação de cada par de objetos foi balanceada de modo que todos foram apresentados o mesmo número de vezes (ou seja, quatro vezes).

Na segunda sessão, foi revertida a relação discriminativa do segundo par de objetos: A2 e B2, de modo que $\mathrm{B} 2$ funcionou como estímulo discriminativo e estava correlacionado ao RB (salaminho). O esquema da sessão foi o mesmo descrito para sessão anterior, ou seja, a apresentação deste par ocorreu por duas tentativas consecutivas, depois foram inseridos os pares A1 e B1 e A3 e B3, totalizando quatro apresentações de cada par. 


\section{Análise de dados}

Os dados foram analisados de acordo com as porcentagens de acertos em cada sessão de linha de base e testes. Além dessa consideração, nas tentativas de testes fez-se uma análise separada priorizando apenas a primeira escolha em relação a cada par de objetos. Este é um tipo importante de resultado, uma vez que os testes foram realizados com a utilização do reforçamento e visaram verificar o quanto os animais eram capazes de generalizar a reversão realizada em um par de objetos, ou seja, a reversão espontânea das funções em dois pares após a reversão de um deles seria um indício de formação de classes entre os três estímulos de cada conjunto.

\section{RESULTADOS}

A Figura 2 apresenta as porcentagens de acertos de cada sujeito do estudo em todas as sessões do experimento. As linhas verticais tracejadas delimitam as sessões conduzidas para avaliar o desempenho dos animais frente aos pares de estímulos mencionados na parte inferior de cada gráfico.

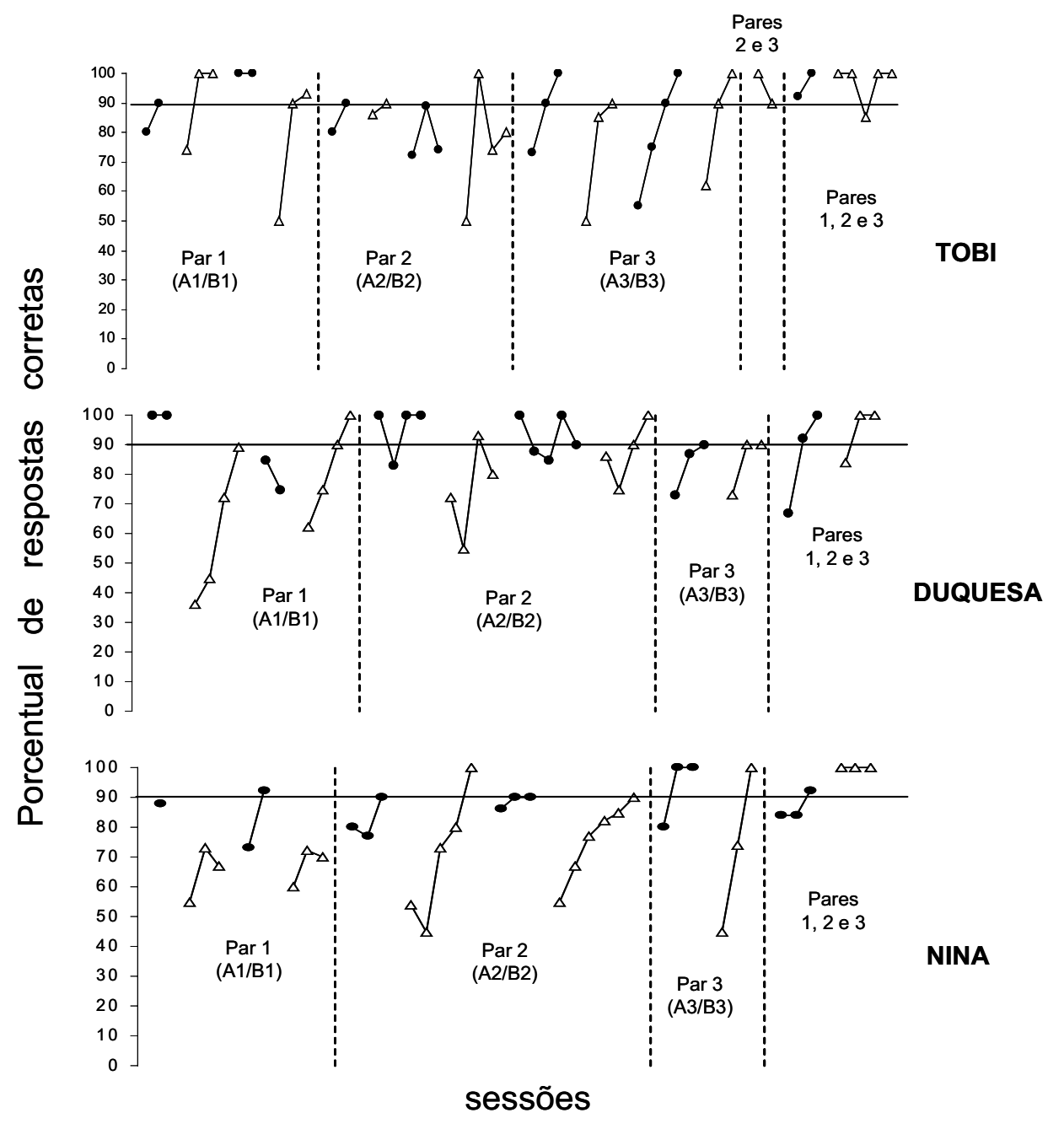

- S+: estímulos $A$ (reforçador A, salsicha)

$\triangle S+$ : estímulos $B$ (reforçador $B$, salaminho)

Figura 2. Desempenho geral dos sujeitos ao longo das sessões experimentais. Cada sinal em destaque nos gráficos acima marca 0 porcentual de respostas corretas em uma sessão. Círculos cheios representam o índice de acertos em sessões que tinham os estímulos do conjunto A como alternativas corretas de escolha; triângulos vazados representam o índice de acertos em sessões que tinham os estímulos do conjunto $\mathrm{B}$ como alternativas corretas de escolha. 
O estabelecimento da linha de base, consistindo de sessões de discriminações simples simultâneas e reversões sucessivas com um par de objetos de cada vez, teve a duração de 33 a 39 sessões. As maiores quedas nos desempenhos durante as sessões de linha de base foram observadas quando as reversões ocorreram do reforçador RA (salsicha) para o reforçador RB (salaminho). Nenhuma tendência em relação à diminuição do número de sessões necessário para atingir o critério de aprendizagem das reversões ou em relação ao aumento constante da porcentagem de acerto em cada primeira sessão, ao longo das sucessivas reversões, pode ser apontada. Também nenhuma queda sistemática no desempenho delas foi observada de forma que pudesse seguramente ser atribuída à introdução de novos pares de estímulos. O que pode ser claramente observado é que nas sessões que envolveram a reversão de RA para $\mathrm{RB}$ as porcentagens de acertos foram mais baixas para todos os animais do que nas sessões onde a reversão ocorreu de RB para RA.

As sessões de teste incluíram tentativas com os três pares de estímulos em apresentações aleatórias. Foram realizadas seis (Duquesa e Nina) ou sete (Tobi) sessões de teste e as porcentagens de acertos de cada sujeito nestas sessões estão apresentadas na Figura 2, à direita, depois da última linha tracejada. Tobi acertou $92 \%$ das tentativas na primeira sessão de teste, $100 \%$ na segunda, terceira, quarta sessões, $85 \%$ na quinta sessão e $100 \%$ nas duas últimas sessões; Duquesa acertou $67 \%$ das tentativas na primeira sessão de teste, $92 \%$ na segunda, $100 \%$ na terceira, $84 \%$ na quarta e $100 \%$ na quinta e sexta sessões; Nina acertou $84 \%$ das tentativas na primeira sessão, $84 \%$ na segunda, $92 \%$ na terceira e $100 \%$ nas demais sessões de teste.

O desempenho de cada sujeito na primeira tentativa que introduzia um novo par de estímulos nas sessões de teste é apresentado na Tabela 1.

TABELA 1

Escolhas Realizadas por Cada Sujeito na Primeira Tentativa em que Foi Apresentado Cada Par de Estímulos.

\begin{tabular}{|c|c|c|c|c|c|c|c|c|c|c|c|c|c|c|c|c|c|c|c|}
\hline & \multicolumn{7}{|c|}{$\begin{array}{c}\text { Tobi } \\
\text { sessões }\end{array}$} & \multicolumn{6}{|c|}{$\begin{array}{l}\text { Duquesa } \\
\text { sessões }\end{array}$} & \multicolumn{6}{|c|}{$\begin{array}{c}\text { Nina } \\
\text { sessões } \\
\end{array}$} \\
\hline & 1 & 2 & 3 & 4 & 5 & 6 & 7 & 1 & 2 & 3 & 4 & 5 & 6 & 1 & 2 & 3 & 4 & 5 & 6 \\
\hline Par 1 & $\sqrt{ }$ & $\sqrt{ }$ & $\sqrt{ }$ & $\sqrt{ }$ & $x$ & $\sqrt{ }$ & $\sqrt{ }$ & $\sqrt{ }$ & $\sqrt{ }$ & $\sqrt{ }$ & $\sqrt{ }$ & $\sqrt{ }$ & $\sqrt{ }$ & $\sqrt{ }$ & $\sqrt{ }$ & $\sqrt{ }$ & $\sqrt{ }$ & $\sqrt{ }$ & $\sqrt{ }$ \\
\hline Par 2 & $\sqrt{ }$ & $\sqrt{ }$ & $\sqrt{ }$ & $\sqrt{ }$ & $\sqrt{ }$ & $\sqrt{ }$ & $\sqrt{ }$ & $\sqrt{ }$ & $X$ & $\sqrt{ }$ & $\sqrt{ }$ & $x$ & $\sqrt{ }$ & $\sqrt{ }$ & $x$ & $\sqrt{ }$ & $\sqrt{ }$ & $\sqrt{ }$ & $\sqrt{ }$ \\
\hline Par 3 & $\sqrt{ }$ & $\sqrt{ }$ & $\sqrt{ }$ & $\sqrt{ }$ & $\sqrt{ }$ & $\sqrt{ }$ & $\sqrt{ }$ & $\sqrt{ }$ & $\sqrt{ }$ & $\sqrt{ }$ & $\sqrt{ }$ & $\sqrt{ }$ & $\sqrt{ }$ & $\sqrt{ }$ & $\sqrt{ }$ & $\sqrt{ }$ & $\sqrt{ }$ & $\sqrt{ }$ & $\sqrt{ }$ \\
\hline
\end{tabular}

Escolhas corretas: $\sqrt{ }$; escolhas incorretas: $X$.

Tobi não fez a escolha correta apenas na primeira tentativa em que foi apresentado o Par 1 na quinta sessão de teste a que foi submetida; Duquesa não acertou ao fazer escolhas diante da primeira apresentação do Par 2 na segunda e quinta sessões; Nina não fez a escolha correta apenas na primeira tentativas em que foi apresentado o Par 2 na segunda sessão de teste.

\section{DISCUSSÃO}

$\mathrm{O}$ delineamento experimental utilizado neste estudo foi baseado em treinos de discriminações simples simultâneas com reversões sucessivas nas funções dos estímulos e uso de reforço específico para cada classe de estímulos que se pretendia estabelecer. Foram utili- zados três pares de objetos que eram treinados isoladamente e depois misturados em sessões de teste. Os desempenhos dos cães nas tarefas de treino e teste foram analisados em termos de formação de classes funcionais de estímulos e learning set. Os resultados obtidos fornecem indícios de que o procedimento utilizado foi eficiente na geração das classes funcionais previstas: os sujeitos apresentaram altas porcentagens de acertos em todas as sessões de teste e, além disso, os registros das primeiras tentativas com cada par de objetos mostraram reversões espontâneas nas suas funções. Esses dados são inéditos com a espécie em questão e foram gerados de forma inovadora em relação a outros estudos que vêm tratando o problema da formação de classes de estímulos com não huma- 
nos, tanto em relação ao procedimento quanto ao tempo de treino necessário para a obtenção de resultados positivos em teste. O procedimento de treino das discriminações simples teve duração de 33 a 39 sessões, mais seis ou sete sessões de testes totalizaram, no máximo, 46 sessões no total, tempo de experimento típico de pesquisas com humanos com desenvolvimento típico, o que torna o procedimento prático $\mathrm{e}$ econômico, ao contrário dos estudos com não humanos citados anteriormente, que necessitaram de anos de treino (inviabilizando, inclusive, testes com espécies cujo tempo de vida é curto, como abelhas).

Os dados obtidos nas sessões de teste também refletiram ocorrências interessantes relacionadas com as reversões espontâneas e o uso de reforço específico para cada classe de estímulos. Na Tabela 3, estão assinaladas com asteriscos as tentativas em que os sujeitos escolheram de acordo com a contingência correta já na primeira tentativa do primeiro par, ou seja, teoricamente, as cachorras "adivinharam" a reversão das funções discriminativas dos estímulos. Esses dados tornam necessário especular acerca de fatores que poderiam influenciar o comportamento dos cães, mas que não foram previstos e controlados experimentalmente. Talvez uma hipótese plausível para explicar esse tipo de responder seja a possibilidade de o reforçador específico para cada classe ter exercido um tipo de controle condicional sobre o responder dos animais. Comumente, estava disponível na sala experimental apenas o item alimentar que seria utilizado naquele dia, correlacionado com um conjunto de estímulos, mas não com o outro. Assim, o responder correto dos animais antes mesmo de qualquer exposição às contingências vigentes naquela sessão estaria condicionado a um controle do tipo: "Se salsicha, então bola branca". O animal teria acesso ao reforçador disponível naquele dia por meio, principalmente, do olfato. A validação dessa hipótese de controle condicional do reforçador sobre o responder do animal carece obviamente, de comprovação empírica.

Uma alternativa para explicação das reversões espontâneas realizadas pelos animais remete a conhecida habilidade dos cães em perceber e responder prontamente às dicas sociais dadas por humanos. Estudos que verificaram essa capacidade discutem que a dependência que esses animais têm dos humanos para obtenção de reforçadores primários é uma ocasião poderosa para que eles sejam extremamente sensíveis em identificar qual a resposta está sendo esperada pelo humano (Udell \& Wynne, 2008). Mesmo considerando todo controle dos pesquisadores para evitar a emis- são de qualquer sinal indicativo da resposta correta (dois observadores, filmagens, treino dos pesquisadores), assume-se que os animais possam ter percebido sinais imperceptíveis para os pesquisadores, como contato do olhar com os estímulos, linguagem corporal, entre outras alternativas. Sugere-se que estudos posteriores controlem ainda mais essa possibilidade, com a inserção de equipamentos para coleta de dados que evitem o contato do animal com o pesquisador, como é o caso do computador (Aust, Range, Steurer, $\&$ Huber, 2008).

A respeito do desenvolvimento de learning set, não foram verificados indícios de que as reversões foram ficando cada vez mais fáceis para os animais. Também aqui, o uso do reforço diferente e específico para cada classe pode ter atuado como uma variável nova em relação a outros estudos da área. Na Tabela 1 é possível verificar que as reversões de salsicha (R1) para salminho (R2) foram mais difíceis para os animais, independente do momento em que a sessão ocorreu no procedimento. Nesse aspecto, o uso dos reforçadores específicos e diferenciais para cada classe parece ter dificultado a formação do learning set, muito possivelmente por diferenças nas preferências dos animais em relação aos itens alimentares. Para o estudo foram escolhidos os dois itens alimentares de maior preferência de acordo com um pré-teste, mas a salsicha parecia ter um valor reforçador bem maior que o salaminho.

O procedimento descrito no presente trabalho pareceu ser adequado e facilitador de resultados positivos e rápidos, passíveis de serem replicados com diferentes espécies, o que o torna bastante promissor para trabalhos futuros. Variações no procedimento como, por exemplo, a não utilização dos reforçadores diferenciais e específicos talvez seja bem-vinda, ou, se forem utilizados os reforçadores específicos, que eles sejam escolhidos na escala mediana de preferência para que a mesma não enviese os dados das reversões, dificultando o desenvolvimento de learning set.

\section{REFERÊNCIAS}

Aust, U., Range, F., Steurer, M., \& Huber, L. (2008). Inferential reasoning by exclusion in pigeons, dogs, and humans. Animal Cognition, 11, 587-597.

De Rose, J. C., McIlvane, W. J., Dube, W. V., Galpin, V. C., \& Stoddard, L. T. (1988). Emergent simple discriminations established by indirect relations to differential consequences. Journal of the Experimental Analysis of Behavior, 50, 1-20.

Goulart, P. R. K., Galvão, O. F., \& Barros, R. S. (2003). Busca de formação de classes de estímulos via procedimento de rever- 
sões repetidas de discriminações simples combinadas em macaco prego (Cebus apella). Interação em Psicologia, 7, 109. 119.

Kastak, C. R., Schusterman, R. J., \& Kastak, D. (2001). Equivalence classification by California sea lions using class-specific reinforcers. Journal of the Experimental Analysis of Behavior, 76, 131-158.

Lionello DeNolf, K. M., Canovas, D. S., de Souza, D.G., Barros, R. S., \& Mcllvane, W. J. (2008). Reversal learning set and contingency class formation in children with and without autism. The Psychological Record, 58, 15-36.

Sidman, M. (1994). Equivalence relations: A research history. Boston, MA: Authors Cooperative.

Sidman, M. (2000). Equivalence relations and the reinforcement contingency. Journal of the Experimental Analysis of Behavior, $74,127-146$
Sidman, M., \& Tailby, W. (1982). Conditional discrimination vs. matching-to-sample: An expansion of the testing paradigm. Journal of the Experimental Analysis of Behavior, 37, 5-22.

Udell, M. A. R., \& Wynne, C. D. L. (2008). A review of domestic dogs' (canis familliaris) human-like behaviors: Or why behavior analysts should stop worrying and love their dogs. Journal of the Experimental Analysis of Behavior, 89, 247-261.

Vaughan Jr., W. (1988). Formation of equivalence sets in pigeons. Journal of Experimental Psychology: Animal Behavior Processes, $14,36-42$.

Sobre os autores:

Recebido: 14/01/2008

Última revisão: 02/10/2008 Aceite final: $10 / 12 / 2008$

Camila Domeniconi: Professora Doutora no Departamento de Psicologia da Universidade Federal de São Carlos.

Renato Bortoloti: Psicólogo, doutor em Teoria e Pesquisa do Comportamento pela Universidade Federal do Pará. Atualmente pesquisador associado do Departamento de Psicologia da Universidade Federal de São Carlos e bolsista de pós doutorado da Fapesp.

Leilane Cristine Krutzfeldt Antoniazzi: Aluna de graduação no curso de Psicologia da UFSCar, iniciação científica voluntária.

Tássia Eid Nogueira Mendes: Aluna de graduação no curso de Psicologia da UFSCar, iniciação científica Propg/UFSCar.

Endereço para correspondência: Camila Domeniconi - Departamento de Psicologia - Universidade Federal de São Carlos - Rod. Washington Luís, km 235 - 13565-905 São Carlos - SP - Endereço eletrônico: camila@ power.ufscar.br. 